\title{
Sequence redundancy under conditions of randomization and spontaneous activity
}

\author{
STEFAN SLAK, JOSEPH I. SHAFFER, and NANCY C. BARONE \\ University of Toledo, Toledo, Ohio 43606
}

\begin{abstract}
The purpose of the study was to compare the sequence redundancy under conditions of randomization with the sequence redundancy under conditions of spontaneous activity. One group was instructed to randomize three alternative push-button responses, and the other group was asked to produce a spontaneous sequence of such responses. Both tasks were self-paced and performed twice. Redundancy up to the fourth order was computed. Completion times and information production rates were determined. Redundancy was significantly higher in the spontaneous activity condition. Information production rate analysis confirmed that there was a greater degree of nonrandomness in the spontaneous activity condition. The effect of test order on redundancy was explained in terms of the difference in completion times between randomization and spontaneous activity conditions. Redundancy was characterized as a possible measure of behavioral stereotypy.
\end{abstract}

It has been demonstrated that human subjects are incapable of generating a totally random sequence (e.g., Slak \& Hirsch, 1974; Slak, Hirsch, \& Syrja, 1979; Warren $\&$ Morin, 1965). As the number of alternatives to be randomized increases, it becomes progressively more difficult to randomize the sequence (Slak \& Hirsch, 1974). There is some evidence that inability to randomize is correlated with neuroticism (Mittenecker, 1960) and psychoticism (Mittenecker, 1960; Yavuz, 1963). The random-number generation task has also been suggested as a possible measure of attention (Evans \& Graham, 1980).

The best measure of randomness is the measure of information provided by information theory. Nonrandomness is measured as informational redundancy. The measure of $n$th order of redundancy $\left(C_{n}\right)$ is obtained from nth-order relative entropy $\left(R_{n}\right)$, so that $C_{n}=1-R_{n}$ and $R_{n}=H_{n} / H_{\max }$, where $H_{n}$ is the estimate of average information, given $\mathrm{n}-1$ known preceding elements, and $\mathrm{H}_{\max }$ is the maximum possible information. (For other computational details, see Attneave, 1959, or Slak, 1977.)

Psotka (1981) refers to behavior such as that required in randomization of responses as "neither very predictable nor random." Indeed, the sequencing behavior can be viewed as occurring somewhere on the continuum between total randomness with zero redundancy and minimum predictability at one extreme and total nonrandomness with redundancy equal to one and complete predictability at the other extreme. Randomization instructions ask the subject to produce a sequence that is as unpredictable as possible. Instead of a sequence

The FORTRAN IV program for redundancy scoring was prepared by Hewen Slak. Requests for reprints should be sent to Stefan Slak, Department of Psychology, University of Toledo, Toledo, Ohio 43606. with zero redundancy, subjects produce a sequence whose redundancy may be somewhere between .10 and .35 (Slak \& Hirsch, 1974). At the other end of the continuum, one could ask subjects to produce a totally predictable sequence with redundancy equal to 1.00 , a trivially easy task. One could devise instructions to produce a sequence of specified alternatives with no indication as to the randomness or predictability of the sequence, that is, instructions that would allow the subject to produce any sequence he wishes. The redundancy of such a sequence could then be compared with the redundancy of a sequence randomized by subjects. It is the purpose of the present study to compare redundancy under conditions of spontaneous activity with the sequence redundancy under conditions of randomization.

\section{METHOD}

Subjects

Twenty eight volunteer undergraduate students of both sexes were randomly assigned to two independent groups of 14 subjects each.

\section{Apparatus}

Three round button switches (Arrowhart Actuator Indicator 83501 ) were placed in a triangular array on a panel $33.5 \mathrm{~cm}$ wide and $28.5 \mathrm{~cm}$ long. The buttons were equidistant from each other and $5 \mathrm{~cm}$ apart. In the center of the triangular button array, there was a black patch with a $2-\mathrm{cm}$ diameter, referred to as the initial position. The panel containing the switches was inclined at an angle of $15 \mathrm{deg}$ on a table $57 \mathrm{~cm}$ high. Subjects were seated facing the apparatus. The buttons were wired to a Sodeco printout counter (Model PO-901) that was set to record the sequence of buttonpushes on a tape. The sequence of buttonpresses was terminated by a Grason-Stadler preset counter (Model 1176) that disconnected the printout counter after the required number of buttonpresses had occurred.

\section{Procedure}

The subject-generated sequence consisted of successive pushbutton responses with the right index finger. The subject had to 
place the finger in the initial position, in the middle, between the three buttons, before the onset of responding and immediately after having pressed one of the three buttons, and he continued to press the buttons at a self-paced rate until $\mathbf{4 0 5}$ buttonpressing responses were counted beyond the 10 practice trials given in conjunction with the instructions. The time in seconds taken to perform 405 responses was recorded with a stopwatch.

Each of the two groups of subjects received a different set of instructions about how to sequence the responses. In the randomization condition, subjects were instructed to push the three alternative buttons in such a way as to form a completely random sequence, with repetitions of any particular button permitted. In the spontaneous activity condition, subjects were instructed to push buttons in any order they wished, with repetitions of any particular button permitted.

In each of the two conditions, a 5-min pause was given after the completion of 405 buttonpressing responses, and then the whole task was repeated (that is, the subject produced another sequence of 405 pushbutton responses at a self-paced rate in the same condition). This procedure yielded a 2 by 2 factorial design, with the factors of performance mode (randomization vs. spontaneous activity) and test order (first vs. second test), with repeated measures on the latter factor.

\section{RESULTS}

With the aid of a FORTRAN program, the sequence redundancy from the first to the fourth order was determined. Figure 1 and Figure 2 show the redundancy as a function of the order of redundancy estimate for both conditions and for the first and second test, respectively. Since the validity of the redundancy estimate increases with the order of redundancy estimate, the fourth order of redundancy $\left(\mathrm{C}_{4}\right)$ was taken as the measure of redundancy.

In the first test, $\mathrm{C}_{4}$ was .236 in the randomization condition and .472 in the spontaneous activity condition, with respective standard deviations of .087 and .197 . In the second test, $\mathrm{C}_{4}$ was .257 in the randomization condition and .549 in the spontaneous condition, with respective standard deviations of .105 and .228 . The redundancy in spontaneous activity was significantly higher $[F(1,1)=18.84, p<.01]$. Redundancy increased significantly in the second test $[\mathrm{F}(1,1)=11.13, \mathrm{p}<.01]$.

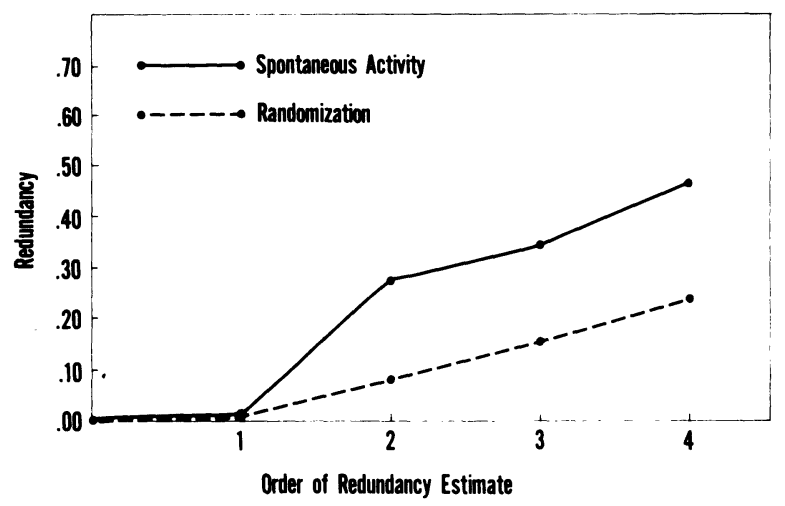

Figure 1. Redundancy in randomization and spontaneous activity conditions as a function of order of redundancy estimate in the first test.

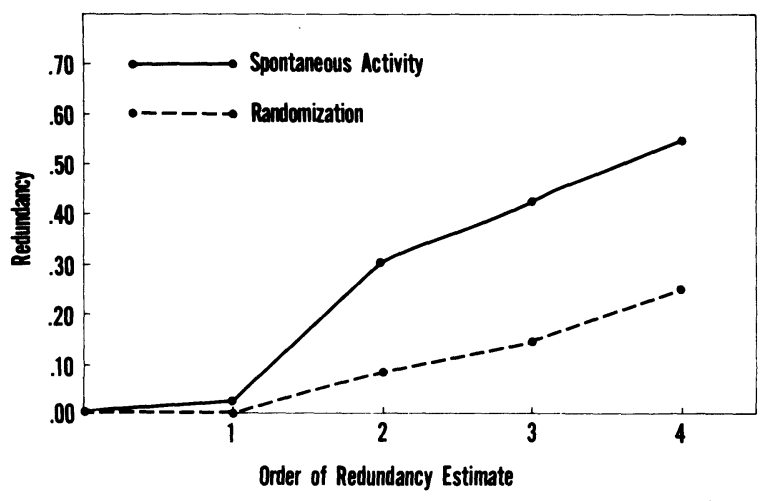

Figure 2. Redundancy in randomization and spontaneous activity conditions as a function of order of redundancy estimate in the second test.

No significant interaction was obtained $[F(1,1)=3.71$, $\mathrm{p}>$.05].

The completion time in the first test was $353 \mathrm{sec}$ in the randomization condition and $303 \mathrm{sec}$ in the spontaneous condition and, in the second test, $309 \mathrm{sec}$ in the randomization condition and $261 \mathrm{sec}$ in the spontaneous condition. The differences between performance modes $[F(1,1)=5.19, p<.05]$ and between the first and the second tests $[F(1,1)=18.53, p<.01]$ were both significant. There was no interaction.

Redundancy in the randomization and spontaneous activity conditions could conceivably be affected by the time it took to complete the task. The data were, therefore, rescored in terms of information production rate (IPR), or the amount of information in bits produced per unit of time (see Slak et al., 1979), defined as $\mathrm{IPR}=405 \mathrm{H}_{4} / \mathrm{t}$ (information in bits per second). Information is reciprocally related to redundancy. One would, therefore, expect from the results of the redundancy analysis that the randomization condition would yield higher IPRs than the spontaneous activity condition would. This was, in fact, the case. In Test 1 , the average IPR was $1.432 \mathrm{bits} / \mathrm{sec}$ for randomization and $1.131 \mathrm{bits} /$ $\mathrm{sec}$ for spontaneous activity. In Test 2 , the respective averages were $1.622 \mathrm{bits} / \mathrm{sec}$ and $1.108 \mathrm{bits} / \mathrm{sec}$. In Test 1 , the standard deviations for randomization and spontaneous activity were .257 and .437 , respectively. In Test 2 , the respective values were .266 and .544 . The difference between the two performance modes was significant $[F(1,1)=8.13, p<.01]$, but the difference between the two successive tests was not $[F(1,1)=3.62$, $p>.05]$. There was a moderate interaction between the two factors $[F(1,1)=5.95, p<.05]$.

Parenthetically, the product-moment correlation coefficient between the first and the second tests was .90 in both randomization and spontaneous activity conditions.

\section{DISCUSSION}

The relevant finding of the study is a rather sizable and highly reliable difference in redundancy between the perfor- 
mance modes of randomization and spontaneous activity. The sequence randomized by subjects yields, roughly speaking, a $25 \%$ redundancy, and the spontaneous sequence displays redundancy of about $50 \%$, which is the midpoint between the minimum and the maximum possible redundancy. It is also in teresting to note that the redundancy of the subject-randomized sequence is halfway between a completely random sequence and a sequence produced by spontaneous activity.

The geometric regularity of these results is likely to be somewhat artificial because of the difference in completion times between the two performance modes. The difference was allowed to occur because the tasks were self-paced. The rationale for the choice of self-paced tasks was the suspicion that pacing of the rate of responding might function as a task superimposed on the main task of sequencing. It is known that a superimposed task has an effect on redundancy of the sequence (Baddeley, 1966) that might also interact with the performance mode. Analysis in terms of IPR showed that the difference between the two performance modes remained significant in spite of the reduction of information production to the same time denominator. Randomization yielded a higher random component than spontaneous activity did, a result consistent with redundancy analysis.

The significant effect of order in the redundancy analysis would seem to suggest that the degree of patterning increases as a function of practice or habit formation. This interpretation, however, is invalidated by the lack of significant order effect in the IPR analysis, suggesting that the increase in redundancy in the second test is due to a decrease in completion time. It is known that an increase in the rate of responding leads to an increase in redundancy, that is, a decrease in information or randomness (Warren \& Morin, 1965).

It is apparent from Figures 1 and 2 that the first-order redundancy is minimal and that an appreciable amount of redundancy builds up beyond the first order. The minimal amount of firstorder redundancy is a consistent finding in randomization studies (e.g., Slak \& Hirsch, 1974). A similar phenomenon seems to be present in spontaneous activity. One should also keep in mind that redundancy curves are essentially cumulative (higher order redundancy always contains lower order redundancy), so that $C_{n} \geqslant C_{n}-1$. For this reason, the analysis of variance was used only on the highest order of redundancy calculated $\left(C_{4}\right)$. Orders of redundancy higher than four would be contaminated with information bias due to the sequence length used.
An interesting way of looking at redundancy is its use as quantification of stereotypy. Indeed, the information measure, either in terms of $\mathrm{H}_{n}$ or $\mathrm{C}_{n}$, may be the best measure of stereotypy, which is characterized hy patterning, repetitiveness of patterning, and reduced variability of responding, because it takes into account all aspects of deviations from randomness. The importance of redundancy as a measure of behavioral stereotypy should, therefore, not be overlooked.

\section{REFERENCES}

Attneave, F. Applications of information theory to psychology: A summary of basic concepts. New York: Holt, 1959.

BADDELEY, A. D. The capacity for generating information by randomization. Quarterly Journal of Experimental Psychology, 1966, 18, 119-129.

Evans, F. J., \& Graham, C. Subjective random number generation and attention deployment during acquisition and overlearning of a notor skill. Bulletin of the Psychonomic Society, 1980, 15, 391-394.

Mittenecker, E. Die informationstheoretische Auswertung des Zeigeversuches bei Psychotichern und Neurotikern. Zeitschrift fur experimentelle und angewandte Psychologie, 1960, 7 , $392-400$.

Psotka, J. Simulating second-guessing in on-line probability learning. Behavior Research Methods \& Instrumentation, 1981, 13, 268-271.

SLAK, S. Informational analysis of the consonant-vowel sequence in Slovene. In Papers in Slovene studies 1976. New York: Society for Slovene Studies, 1977.

SlaK, S., \& Hirsch, K. A. Human ability to randomize sequences as a function of information per item. Bulletin of the Psychonomic Society, 1974, 6, 29-30.

Slak, S., Hirsch, K. A., \& Syrja, K. V. Randomization of univariate and bivariate sequences and Baddeley's limited capacity hypothesis. Journal of Psychology, 1979, 103, 169-182.

W ARren, P. A., \& Morin, R. E. Random generation: Number of symbols to be randomized and the time per response. Psychonomic Science, 1965, 3, 557-558.

YAvUz, H. S. The production of random letters sequences in schizophrenics. Journal of Psychology, 1963, 56, 171-173.

(Received for publication March 1, 1982.) 